TRANSFER REACTIONS AND DEUTERON INDUCED CHARGE-EXCHANGE REACTIONS AT LOW AND INTERMEDIATE ENERGIES

\author{
H. SAKAI \\ Department of Physics, University of Tokyo, Hongo 7-3-1, Bunkyo-ku, \\ Tokyo 113, Japan
}

In this talk I will discuss two subjects which are studied by nuclear reactions with polarized beams at low and intermediate energies. In the first part I will briefly review the present status of the research of the transfer reactions. In the second part I will show the new development of deuteron induced charge-exchange reaction, namely $\left(\vec{d}, d_{s=0}\right)$ and $\left(\vec{d}^{2} \mathrm{He}\right)$ reactions. I will try to show that analyzing power measurements appear to be useful in assigning the Gamow-Teller type transitions.

\title{
1. Transfer reactions
}

Transfer reactions are still of considerable interest despite their long history. For more than 35 years, measurements of transfer reactions have provided useful spectroscopic information. After a polarized beam became available about 25 years ago they became even more important in the spectroscopic studies due to the $j$-dependence in analyzing power and in the reaction theories. Now, with better polarized ion source and measuring techniques, measurements of polarization transfer coefficients are adding new dimensions to our knowledge of the transfer reactions.

Since the 1985 symposium in Osaka about 70 papers on the transfer reaction studies with polarized beams have been published. They can be roughly classified into three groups; spectroscopic studies, D-state problems and reaction mechanisms. It is clearly impossible to discuss each of many interesting results appeared instead I have selected three topics from each categories based on my personal taste.

\subsection{Occupation Probability}

The occupation probability of the single particle state is one of the most fundamental quantity in nuclear physics. The one nucleon transfer reaction is a powerful tool to investigate the single particle states. In particular the study with the polarized beam is very important because of the ability of the assignment of the total spin $j$ by using the vector analyzing power. 
It has recently argued/1/ for the doubly closed-shell nucleus ${ }^{208} \mathrm{~Pb}$ that short-range and tensor correlations give rise to a depletion of normally completely filled orbitals close to the Fermi surface. Estimates of this depletion are as large as $30 \%$, although the possibility of the double counting in the theory is pointed out for this large depletion $/ 2 /$. Even larger depletion of the occupation probability near the Fermi surface have been derived experimentally by the $\left(e, e^{\prime} p\right)$ reaction which is considered to be less ambiguous.

In order to understand this depletion mechanism quantitatively one must look for the missing strength $\left(S_{j}\right)$ in the highly excited region. Because of the high level density of the highly excited region the missing strength is spread over many underlying states. This spreading mechanism is not understood well. To identify such a small piece of strength under the circumstances of high level density a high energy resolution measurement is crucial. The excitation energy beyond the particle unbound, particularly neutron unbound region in heavy nuclei, analyses suffer from the uncertainty of the unbound form factor and at the same time owing to the continuum nature of the states the subtraction of the physical background is required and this subtraction is also the subject of another source of ambiguity.

The occupation probability $n_{j}$ may be defined in the transfer reaction as:

$$
\begin{array}{ll}
n_{j}=S_{j} /(2 j-1) & \text {; pickup reaction, } \\
n_{j}=1-S j & \text {; stripping reaction, }
\end{array}
$$

where $S_{j}$ is the spectroscopic factor derived from the exact finite-range Distorted Wave Born Approximation(DWBA) analysis. The absolute $S_{j}$ value has to be extracted through the analysis. To minimize ambiguities in the course of the analysis care has to be taken in the choice:(i) of bound state wave functions, since $S_{j}$ depends strongly on the surface region where the transfer reaction occurs, and (ii) of the entrance and exit channel optical potentials since they are non-unique.

The occupation probability around ${ }^{40} \mathrm{Ca}$ is one of the most extensively studied nucleus with polarized beams via the pickup and stripping reactions. Here I show some of the recent results around ${ }^{40} \mathrm{Ca}$ nucleus.

i) Particle states via the stripping reaction.

Eckle et al. $3,4 /$ have measured the ${ }^{40} \mathrm{Ca}(\vec{d}, p){ }^{41} \mathrm{Ca}$ reaction at $E_{d}=20 \mathrm{MeV}$ with an overall energy resolutions of $6 \mathrm{keV}$ and found 183 levels up to $8.7 \mathrm{MeV}$ excitation energy. Angular distributions of each level of cross section and vector analyzing power are analyzed in terms of DWBA as well as CCBA calculations with empirical form factors of well depth method to extract absolute $S_{j}$. The obtained results after summing up the spectroscopic factors up to $8.7 \mathrm{MeV}$ excitation energy are listed in Table 1 together with those obtained at $E_{d}=56 \mathrm{MeV}$ by Matoba et al./5/ where the summation is made up to $10 \mathrm{MeV}$ excitation energy. It is very important to investigate the same nucleus by different bombarding energies in order to see the consistency of results.

ii) Hole states via the pickup reaction.

Hole states in ${ }^{39} \mathrm{Ca}$ have been measured via ${ }^{40} \mathrm{Ca}(\vec{p}, d)$ reaction by Matoba et al./6/ at $E_{p}=65 \mathrm{MeV}$ with energy resolution of $30 \mathrm{keV}$ by using the high resolution spectrograph RAIDEN at Research Center for Nuclear Physics(RCNP), Osaka University/7/. 
Observed analyzing power shows clear $j$-dependence and its angular dependence is stable up to the $10 \mathrm{MeV}$ excitation energy. Results are also shown in Table 1.

iii) Occupation probability via the $\left(e, e^{\prime}\right)$ scattering.

Platchkov et al. $/ 8 /$ have performed the $180^{\circ}$ electron scattering experiment on the radioactive target ${ }^{41} \mathrm{Ca}$. Obtained occupation probability of the ground state $1 f_{7 / 2}$ neutron is 0.17 which should be compared to the stripping reaction of Eckle's result of 0.15 or Matoba's $0.24(9)$ as is shown in Table 1 , respectively.

iv)Theoretical prediction.

In the table 1 theoretical predictions on the occupation probability by Nishizaki et al./9/ based on the extended second RPA method are also listed. The ground state correlations due to $2 p-2 h$ states is taken into account and the density dependence is also included in an approximate way. Calculations show very small depletion even for the state close to the Fermi-level. Note that the calculations are for the ${ }^{40} \mathrm{Ca}$ but neither ${ }^{39} \mathrm{Ca}$ nor ${ }^{41} \mathrm{Ca}$. Other theoretical studies predict same magnitudes of depletion/10/.

It is clear from the Table 1 that occupation probabilities deduced from the $(d, p)$ and $(p, d)$ reactions are consistent with each other within statistical errors. Moreover they are also consistent with the result obtained by the ${ }^{41} \mathrm{Ca}\left(e, e^{\prime}\right)^{41} \mathrm{Ca}$ reaction as for the ground state of $1 f_{7 / 2}$. Therefore there seems to be no large disagreement between transfer data and electron scattering datum. In addition, theoretically estimated occupation probabilities agree fairly well with the experimental results. Thus it indicates that the depletion factor larger than 0.3 for the states near the Fermi-level which are predicted in the ref/1/ seems to be very questionable based on the present results.

Table 1: Occupation probabilities around ${ }^{40} \mathrm{Ca}$

\begin{tabular}{|c||c|c|c|c|c|c|}
\hline \hline \multirow{2}{*}{ state } & \multicolumn{2}{|c|}{${ }^{40} \mathrm{Ca}(\vec{d}, p)^{41} \mathrm{Ca}$} & ${ }^{40} \mathrm{Ca}(\vec{p}, d)^{39} \mathrm{Ca}$ & ${ }^{41} \mathrm{Ca}\left(e, e^{\prime}\right)$ & $\begin{array}{c}\text { extended } \\
\text { 20 }\end{array}$ & $\begin{array}{c}{ }^{40} \mathrm{Ca}\left(e, e^{\prime} p\right) \\
{ }^{39} \mathrm{CeV}\end{array}$ \\
\hline $1 d_{5 / 2}$ & 0.88 & $56 \mathrm{MeV}$ & $65 \mathrm{MeV}$ & & & \\
$2 s_{1 / 2}$ & 0.97 & $0.71(25)$ & $0.82(8)$ & & 0.92 & $0.83(6)$ \\
$1 d_{3 / 2}$ & 0.83 & $0.79(9)$ & $0.93(3)$ & & 0.88 & $0.64(6)$ \\
$1 f_{7 / 2}$ & 0.00 & $0.24(9)$ & $0.04(4)$ & & 0.91 & $0.65(7)$ \\
$g n d$ & 0.15 & $0.24(9)$ & $0.04(4)$ & 0.17 & & \\
$2 p_{3 / 2}$ & 0.22 & $0.10(9)$ & & & 0.07 & $0.11(1)$ \\
$1 f_{5 / 2}$ & 0.00 & $0.02(14)$ & & & 0.03 & \\
$2 p_{3 / 2}$ & 0.22 & $0.01(11)$ & & & 0.03 & \\
\hline$E_{x}$ range & & & & & & \\
$(\mathrm{MeV})$ & $0-8.7$ & $0-10$ & $0-10$ & & & $0-22$ \\
\hline reference & $/ 3,4 /$ & $/ 5 /$ & $/ 6 /$ & $/ 8 /$ & $/ 9 /$ & $/ 11,12 /$ \\
\hline \hline
\end{tabular}

gnd indicates the occupation probability pertinent to the $1 f_{7 / 2}$ ground state in ${ }^{41} \mathrm{Ca}$. 
However, a large disagreement between the transfer results and the electron results is known as for the proton occupation probability. In Table 1 occupation probabilities for the proton orbits deduced from the proton knockout reaction ${ }^{40} \mathrm{Ca}\left(e, e^{\prime} p\right)^{39} \mathrm{~K}$ $/ 11,12 /$ are also included to compare with transfer results. The occupation probabilities deduced by the $\left(e, e^{\prime} p\right)$ reaction are around 0.65 and substantially smaller for the states around the Fermi-level than those by the $(p, d)$ reaction. Such a large difference can not be explained by the possible difference between proton and neutron states. Kramer et al./11,12/ looked into the origin of this large spectroscopic factor difference by examining the ${ }^{48} \mathrm{Ca}\left(e, e^{\prime} p\right)^{47} \mathrm{~K}$ and ${ }^{48} \mathrm{Ca}\left(\mathrm{d},{ }^{3} \mathrm{He}\right){ }^{47} \mathrm{~K}$ reactions leading to the same final states thus the direct comparison is possible. He claims that if the ${ }^{48} \mathrm{Ca}\left(d,{ }^{3} \mathrm{He}\right)$ data are analyzed with the bound state wave functions which are obtained from $\left(e, e^{\prime} p\right)$ experiments good agreements can be obtained between the spectroscopic factors deduced from both reactions. However the rms radius of the bound state wave functions determined by the $\left(e, e^{t} p\right)$ experiments is larger than that of commonly used in the $\left(d,{ }^{3} \mathrm{He}\right)$ analysis. Kramer's approach is very interesting but it is not clear at this moment whether his approach is appropriate or not. Another disagreement is also reported in proton occupation probabilities, particularly $3 s_{1 / 2}$ orbit, in lead isotopes $/ 13,14,15 /$. It is extremely important to resolve the discrepancy between the hadronic probes and the electron scatterings. Systematic studies by using various probes and reactions are certainly helpful to clarify.

1.2 Polarization Transfer Coefficients and Deuteron D-state Amplitude

Polarization transfer coefficients(PTC) act as an amplifier of small matrix elements. There is a chance to pickup small amplitudes such as a tensor part by choosing proper combinations of PTCs. The Invariant Amplitude Method(IAM) proposed by Tanifuji and Yazaki/16/ is a convenient means to investigate relations among the amplitudes. The $(\vec{d}, \vec{p})$ reaction with $\ell_{n}=0$ on the spin $0^{+}$target has 8 PTCs which depend on the tensor interaction out of $12 \mathrm{PTC}$ observables. The tensor interaction is closely related with the deuteron $D$-state. Sagara/17/ found an interesting combination which depends solely on the tensor amplitude $T$ :

$$
\begin{gathered}
a b s . T_{\alpha}=\sigma \sqrt{\left(2 A_{x z}\right)^{2}+\left(K_{x x}^{y}-K_{x z}^{y}\right)^{2}}, \\
2 A_{x z} \propto \operatorname{Re}(T \cdot U), \\
K_{x x}^{y}-K_{z x}^{y} \propto \operatorname{Im}(T \cdot U),
\end{gathered}
$$

where $U$ is the amplitude due to central interaction. He found another similar relation but it is not shown here for brevity. For detail see ref.17. Here the $2 A_{x z}$ and $K_{x x}^{y}-K_{z z}^{y}$ are the real and imaginary part of the product of $T \cdot U$, respectively.

Sagara et al./17,18/ have measured the PTCs $\left(K_{x x}^{y}\right.$ and $\left.K_{z z}^{y}\right)$ and the tensor analyzing power $\left(A_{x z}\right)$ for the ${ }^{28} \mathrm{Si}(\vec{d}, \vec{p})^{29} \mathrm{Si}$ reaction at $18 \mathrm{MeV}$ and obtained abs. $T_{\alpha}$. It is clearly demonstrated that the abs. $T_{\alpha}$ depends strongly on the amplitude of the tensor part alone as expected and depends very weakly on the change of the optical potentials. On the other hand $A_{x z}$ and $K_{x x}^{y}-K_{z x}^{y}$ do depend strongly on the optical potentials and only 10 $\%$ change of the central part of the optical potential give rise to a sizable change. The 
$a b s . T_{\alpha}$ is sensitive to the whole angular range. This means that the D-state amplitude can be extracted from the most stable region where the cross section is large. The $a b s . T_{\alpha}$ is nicely reproduced with a D-state amplitude of Reid/19/ which corresponds to the $\mathrm{D}$-state probability of $6.5 \%$. The $K_{y}^{y^{\prime}}$ of the $(\vec{d}, \vec{p})$ reaction is often used to get the deuteron D-state effect. Unfortunately it is most sensitive not only at the minimum of the differential cross section but also to a choice of optical potentials and reaction mechanisms.

Sagara et al. extended the measurements to the $\ell_{n} \neq 0$ transitions in ${ }^{40} \mathrm{Ca}$ and ${ }^{52} \mathrm{Cr}(\vec{d}, \vec{p})$ reactions and found a similar sensitivity to the deuteron $\mathrm{D}$-state amplitude. It has been pointed out by Tanifuji that a similar relation also holds for the $\ell_{n} \neq 0$ transition in terms of the DWBA formalism $/ 20 \%$.

As is clear from this example that the PTC is a useful tool to investigate small amplitudes which are otherwise masked.

$1.3(d, p)$ reactions and the adiabatic approach

The coupled discretized continuum channels (CDCC) approach which provides accurate three-body wave functions in the deuteron nucleus system has been developed by Kyushu group/21/. In the transfer reaction at medium energies both spin-dependent distortion and D-state effects are important. However these effects have not yet been incorporated in the CDCC because of increased complexity. As a result the adiabatic distorted wave approximation (ADWA) is often employed for the analysis of the transfer reactions.

It has been suggested that the $(d, p)$ reaction associated with a large $\ell_{n}$ transfer at intermediate energies is dominated by the far-side amplitudes $/ 22 /$. Experimentally a signature has been seen as the disappearance of the interference pattern from the cross sections for the ${ }^{116} \mathrm{Sn}(\vec{d}, p)^{117} \mathrm{Sn}$ reactions at $79 \mathrm{MeV}$ as $\ell_{n}$ increases/23,24/. Cross sections and analyzing powers are analysed with ADWA calculations. Unexpectedly the well matched transitions such as $\ell_{n}=4$ where the far-side amplitudes dominate is poorly described by the ADWA instead the miss matched transition like $\ell_{n}=0$ where the farand near-side amplitudes interfere is well described. The shortcoming of the ADWA treatment has become clear through these analyses $/ 25 /$.

In the case of $j_{n}=\ell_{n}-1 / 2$ transitions above shortcoming becomes most apparent. Johnson has shown that the far-side dominance together with the parity conservation leads to the following relation between polarization observables $/ 22 /$;

$$
3 P_{y}+2 A_{y y}+1=0
$$

where $P_{y}$ is the outgoing proton polarization and $A_{y y}$ is the deuteron tensor analyzing power. This relation has been examined by using the well matched transition $\ell_{n}=3$ in the reaction of ${ }^{86} \mathrm{Zn}(\vec{d}, p)^{67} \mathrm{Zn}\left(j^{\pi}=\frac{5^{-}}{2}\right)$ at $88 \mathrm{MeV} / 26 /$. The $P_{y}$ is obtained from the equivalent analyzing power in the time-reversed ${ }^{67} \mathrm{Zn}(\vec{p}, d)^{66} \mathrm{Zn}$ reaction at $92 \mathrm{MeV}$. It is found that an interference pattern is present in the difference function which is defined as $P_{y}+\left(1+2 A_{y y}\right) / 3$. The ADWA calculations which include both S- and D-state stripping in finite range and is corrected for non-local effects failed to reproduce this oscillating pattern. Note that the difference function is insensitive to spin-orbit interactions or spinflip effects in the deuteron channel. The oscillation in the difference function indicates 
the interference of near- and far-sides amplitudes. For that the near-side amplitude has to be increased to an extent as much as comparable to the far-side amplitude by some reason.

An improvement of the ADWA has been attempted by employing an approach of the quasi-adiabatic approximation of Amakawa, Austern and Vincent/27/. In the ADWA treatment the breakup states of the incident deuteron is degenerated in energy into the ground state of deuteron while in the quasi-ADWA treatment this degeneracy is removed and the center-of-mass energy of breakup $n p$ pair is explicitly taken into account. With this prescription the interference is much better reproduced in spite of the preliminary quasi-ADWA calculations with zero-range approximation/26/. Thus the quasi-ADWA treatment is able to recover the missing near-side amplitudes. This is a very encouraging result and the refinement of the calculation including the finite-range should be done. Such an effort is valuable to understand the three-body reaction mechanism in a simpler model. At the same time it is obviously needed to pursuit the CDCC calculations which describe the three-body reaction accurately to assess the validity of the quasi-ADWA treatment as well.

\section{Deuteron induced charge-exchange reactions}

The study of the spin-isospin excitations in nuclei is currently of considerable interest/27/. The $\left(d, d_{s=0}\right)$ and $\left(d,{ }^{2} \mathrm{He}\right)$ reactions are, in many respects, a unique tool for exciting spin- and isospin-flip states, since both reactions excite exclusively spin- and isospin-flip states. $d_{s=0}$ or ${ }^{2} \mathrm{He}$ indicates a proton-neutron or proton-proton system coupled to the singlet $S$-state $\left[{ }^{1} S_{0}\right]$. Tensor analyzing power measurements in the reactions of $\left(\vec{d}, d_{s=0}\right)$ and $\left(\vec{d}^{2}{ }^{2} \mathrm{He}\right)$ give almost identical information as obtained through the polarization transfer measurements in the $\left(\vec{p}, \overrightarrow{p^{\prime}}\right)$ and $(\vec{n}, \vec{p})$ reactions which require elaborate double or triple scattering measurements/28/. Here, recent new development of the deuteron induced charge-exchange reactions is described. Some emphasis is placed on the $\left(\vec{d}, d_{s=0}\right)$ rection since such a reaction has never been studied before partly because of a serious background from the elastic breakup of the deuteron and partly because a low counting efficiency of neutrons. Significant progress in spin-isospin response functions for the $\Delta$ and quasi- free scattering region has been made at SATURNE by the $\left(\vec{d},{ }^{2} \mathrm{He}\right)$ reaction and results is presented by Delorme in this conference/29/.

\subsection{Migdal-Watson approximation}

Migdal/30/ and Watson/31/ have shown that the three-body reaction problem can be reduced to the two-body reaction problem, which is certainly much easier to handle, under the assumptions of: small relative energy of $p n$ or $p p$ pair, very strong final state interaction(FSI) and short range character of reaction interactions. The triple differential cross section for the $\left(\vec{d}, d_{s=0}\right)$ reaction is ,

$$
\frac{d^{3} \sigma}{d \Omega_{A-p n} d \Omega_{p n} d \epsilon_{p n}} \propto P\left(\epsilon_{p n}\right)
$$




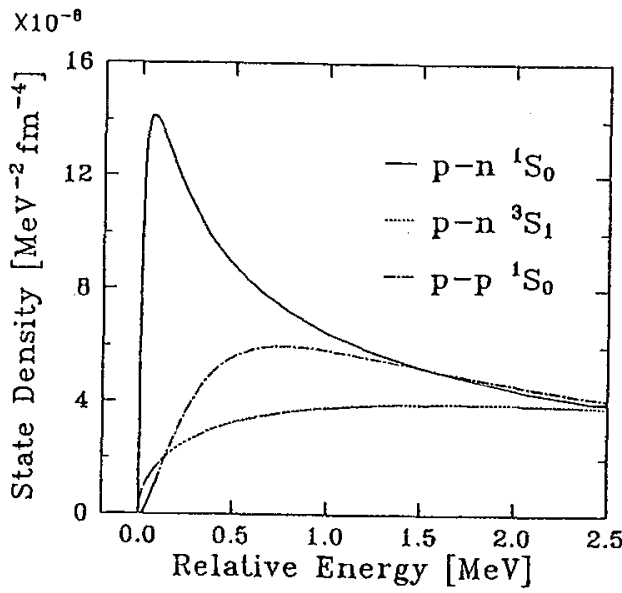

Figure 1: State density as a function of relative energy of $p n$ or $p p$ pair based on the Migdal-Watson theory.

where $P\left(\epsilon_{p n}\right)$ is the state density and $\epsilon_{p n}$ is the relative energy of the $p n$ system. Extension to the $\left(d,{ }^{2} \mathrm{He}\right)$ rection is obvious. Figure 1 shows the state density $P(\epsilon)$ for the $p n$ or $p p$ pair as a function of relative energies of them. The ${ }^{1} S_{0}$ state of the $p n$ pair has a sharp peak at about $60 \mathrm{keV}$ while that of $p p$ pair shows a broad bump peaked at about $700 \mathrm{keV}$. In the $p n$ scattering the ${ }^{3} S_{1}$ state is allowed while in the $p p$ scattering it is strictly forbidden due to the Pauli principle. The contribution from the ${ }^{3} S_{1}$ state in the $(d, p n)$ reaction can be removed experimentally by utilizing a large difference in the shape of $P(\epsilon)$ between ${ }^{1} S_{0}$ state and ${ }^{3} S_{1}$ state.

Although the Migdal-Watson theory is able to describe conveniently the relative energy distribution, it is not possible to give an absolute magnitude or an angular distribution of the cross sections. In order to calculate these quantities the DWBA treatment under the strong FSI has been developed by Okamura/32/ by extending the conventional DWBA code for the two-body reaction although it is primarily a three-body reaction problem.

\section{$2.2^{12} \mathrm{C}\left(\vec{d}, d_{s=0}\right)$ and ${ }^{12} \mathrm{C}\left(\vec{d},{ }^{2} \mathrm{He}\right)$ reactions}

The reaction mechanism has been studied by using ${ }^{12} \mathrm{C}$ target whose structure is well known. Both ${ }^{12} \mathrm{C}\left(\vec{d}, d_{s=0}\right)$ and ${ }^{12} \mathrm{C}\left(\vec{d},{ }^{2} \mathrm{He}\right)$ reactions is able to excite the final states in analogue relations. Experimental procedures are described elsewhere/32,33,34/ and therefore they will be described briefly. All the measurements were performed at RCNP, Osaka University. Polarized deuteron beams of 56 and $70 \mathrm{MeV}$ with $80 \%$ polarization of the ideal value were provided by the AVF cyclotron.

i) ${ }^{12} \mathrm{C}\left(\vec{d}, d_{s=0}\right){ }^{12} \mathrm{C}$ measurement $/ 32,33 /$

Schematic layout of the experimental setup is shown in Fig.2(a). Protons were detected by a counter telescope which consisted of a plastic scintillation counter and $\Delta E$ $E$ silicon solid state detectors. Neutrons were detected by a NE213 liquid scintillation counter and their energies were determined by the time-of-flight method. The angular distribution from $15^{\circ}$ to $65^{\circ}$ was measured simultaneously by using six pairs of counter 

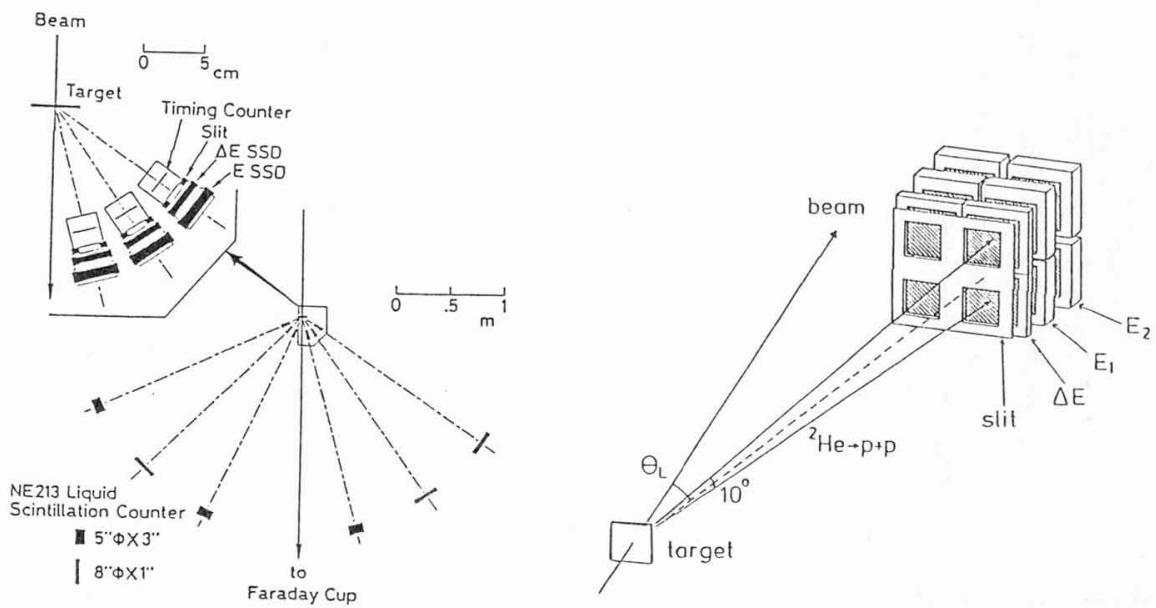

Figure 2: Schematic layout of the experimental setup for the $p n$ coincidence measurement(left side) and for the $p p$ coincidence measurement(right side).

telescopes. Typical solid angles were 15 and $8 \mathrm{msr}$ for the proton and neutron detectors, respectively. Measurement of $\theta=0^{\circ}$ was made with the spectrograph DUMAS/35/. Fig. 3 shows a two-dimensional coincidence energy spectrum at $\theta_{p}=\theta_{n}=35^{\circ}$ for the ${ }^{12} \mathrm{C}(d, p n)$ reaction at $56 \mathrm{MeV}$. A corresponding summed energy spectrum is shown in fig.4a. The well-known $T=1$ states of ${ }^{12} \mathrm{C}$ are strongly excited showing a clear selectivity of spin- and isospin-flip mode. The $T=0$ state such as the ground and $2^{+}(4.4 \mathrm{MeV})$ states are also excited due to the contribution from the ${ }^{3} S_{1}$ state. Owing to its narrow distribution, unambiguous identification of the ${ }^{1} S_{0}$ state is made by fitting the relative energy spectrum $\epsilon_{p n}$ with the Migdal-Watson theory, taking the detection efficiency of the neutron detector and the geometrical solid angles of the proton and neutron detector into account, as shown in fig. $4 \mathrm{~b}$.

The differential cross sections and vector analyzing powers of the ${ }^{12} \mathrm{C}\left(\vec{d}, d_{s=0}\right){ }^{12} \mathrm{C}$ reaction are shown in fig.5, for the unresolved $1^{+}$and $2^{+}$states, the $2^{-}-4^{-}$doublet and $1^{-}$state. The solid curves in the figure are the results of the prior-form DWBA analysis without any normalization. The experimental angular distributions are reasonably well reproduced $/ \mathbf{3 3} /$.

ii) ${ }^{12} \mathrm{C}\left(\vec{d}^{2}, \mathrm{He}\right){ }^{12} \mathrm{~B}$ measurement $/ 34,36,37$ /

Schematic layout of the the silicon counter array for the $p$ - $\left[{ }^{1} S_{0}\right]$ detection is shown in Fig.2(b)/36/. This array structure increases total detection efficiency by about a factor of 5 compared to the case of using only two telescopes because six different pairs of telescopes can be used: four pairs with large efficiency and two smaller efficiency( diagonal pair). Each telescope consists of $\triangle E-E-E_{v}$ SSDs with a solid angle of $13 \mathrm{msr}$. Measurement of $\theta=0^{\circ}$ was made again with the spectrograph DUMAS/35/.

The ${ }^{12} \mathrm{C}\left(\vec{d},{ }^{2} \mathrm{He}\right)^{12} \mathrm{~B}$ reaction was measured at $70 \mathrm{MeV} / 34,37 /$. Results are not shown 


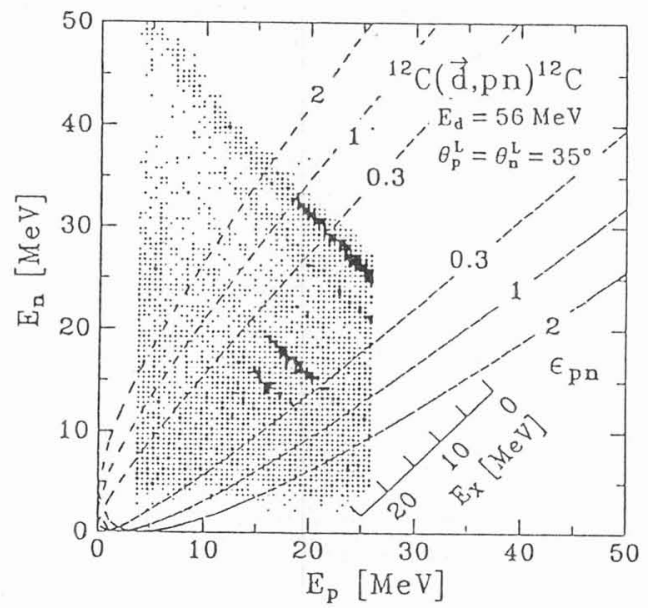

Figure 3: Two-dimensional energy spectrum. Contours of fixed $\epsilon_{p n}$ in $\mathrm{MeV}$ are indicated by dashed curves in the figure.
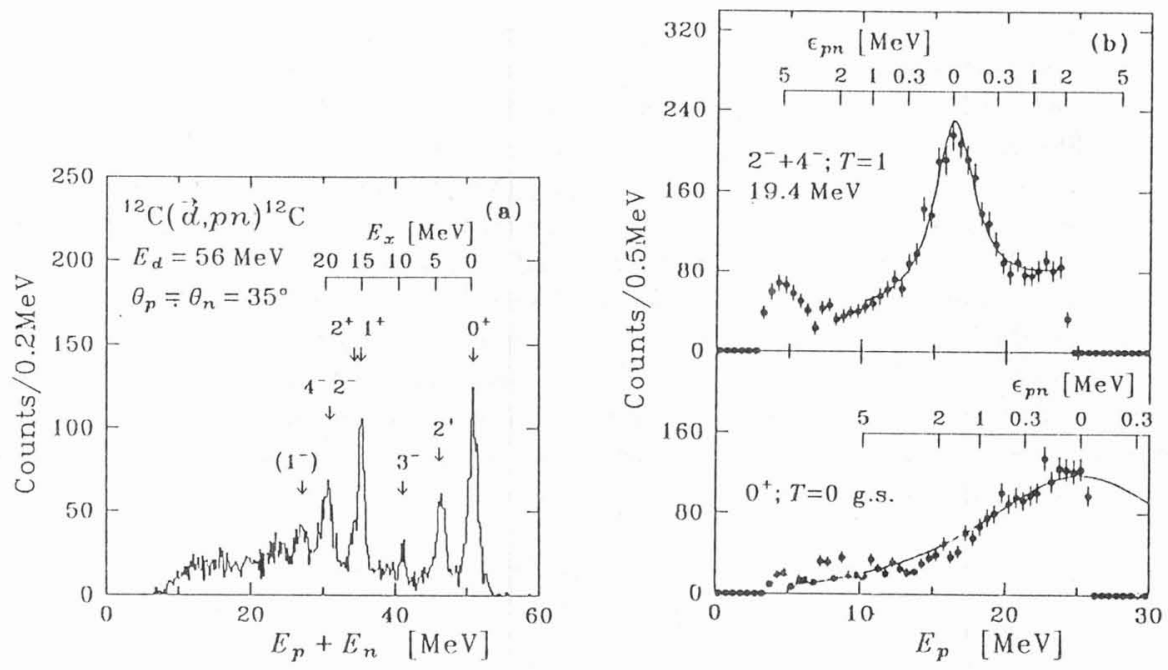

Figure 4: (a) A summed energy spectrum of the protons and neutrons detected at $\theta_{p}=$ $\theta_{n}=35^{\circ}$ from the ${ }^{12} \mathrm{C}(\vec{d}, p n){ }^{12} \mathrm{C}$ reaction at $E_{d}=56 \mathrm{MeV}$. (b) The proton energy spectra corresponding to the $2^{-}-4^{-}$doublet $(T=1)$ and to the ground state $(T=0)$. The solid curves are the fit to the data based on the Migdal-Watson theory. 

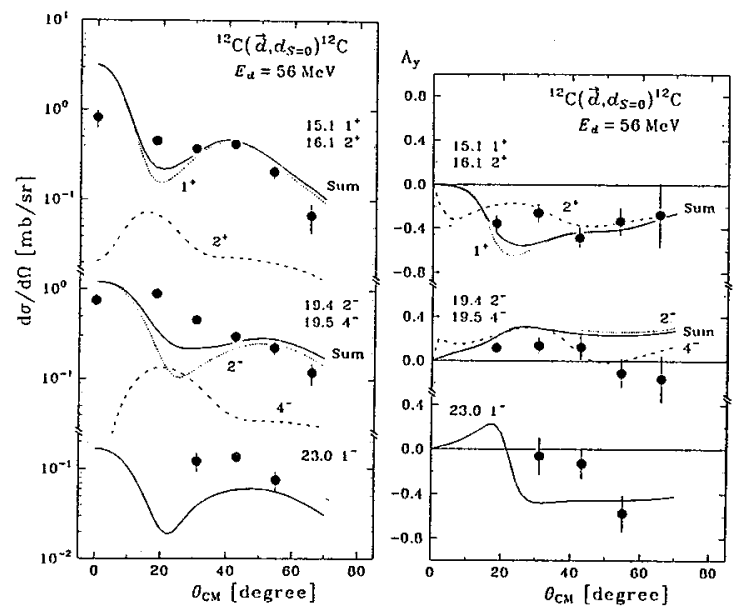

Figure 5: Differential cross sections and vector analyzing powers for the ${ }^{12} \mathrm{C}\left(\vec{d}, d_{s=0}\right)^{12} \mathrm{C}$ reaction. The curves are the results of DWBA calculations.

here. This reaction excites the states which are the parent analogues of the $T=1$ states excited by the ${ }^{12} \mathrm{C}\left(\vec{d}, d_{s=0}\right){ }^{12} \mathrm{C}$ reaction. Obtained angular distributions and analyzing powers are almost identical to those of the ${ }^{12} \mathrm{C}\left(\vec{d}, d_{s=0}\right)^{12} \mathrm{C}$ reaction if they are scaled by the momentum transfer instead of the scattering angle.

This similarity between ${ }^{12} \mathrm{C}\left(\vec{d}, d_{s=0}\right){ }^{12} \mathrm{C}$ and ${ }^{12} \mathrm{C}\left(\vec{d}^{2} \mathrm{He}\right){ }^{12} \mathrm{~B}$ reactions strongly suggests that the reactions are dominated by the single-step process. If two- or multi-step processes are important, resultant angular distributions and analyzing powers will be very different since possible intermediate states are very different. It also suggests that the optical potentials of the exit channel for $d_{s=0}$ and ${ }^{2} \mathrm{He}$ are similar. It therefore implies that the unbound objects $d_{s=0}$ and ${ }^{2} \mathrm{He}$ can be regarded as "particle"s. This is the basis of the validity of the present DWBA treatment with FSI.

Another important conclusion can be drawn from the experimental results. The vector analyzing power $\left(A_{y}\right)$ shows a characteristic feature depending on the spin- and parity-transfer: $A_{y}$ is negative for the $1^{+}$state while it is positive for $2^{-}$state at forward angles. This feature is also confirmed by the DWBA calculations and it is insensitive to the parameters used in the calculations. Thus the $A_{y}$ is useful for the spin and parity assignment particularly for the Gamow-Teller transition.

Measurements have been extended to the following targets:

$$
\begin{aligned}
& \left(\vec{d}, d_{s=0}\right) \text { reaction } \ldots{ }^{2} \mathrm{H} / 38 /,{ }^{58} \mathrm{Ni} / 33 / . \\
& \left(\vec{d},{ }^{2} \mathrm{He}\right) \text { reaction } \ldots{ }^{1} \mathrm{H} / 39 /,{ }^{4} \mathrm{He} / 32 /,{ }^{9} \mathrm{Be},{ }^{11} \mathrm{~B} / 40 /,{ }^{13} \mathrm{C} / 34 /,{ }^{14} \mathrm{~N} / 32,34 /,{ }^{16} \mathrm{O},{ }^{40} \mathrm{Ca} / 40 / .
\end{aligned}
$$

In the next section most recent result on the ${ }^{11} \mathrm{~B}\left(\vec{d},{ }^{2} \mathrm{He}\right){ }^{11} \mathrm{Be}$ reaction is shown. 


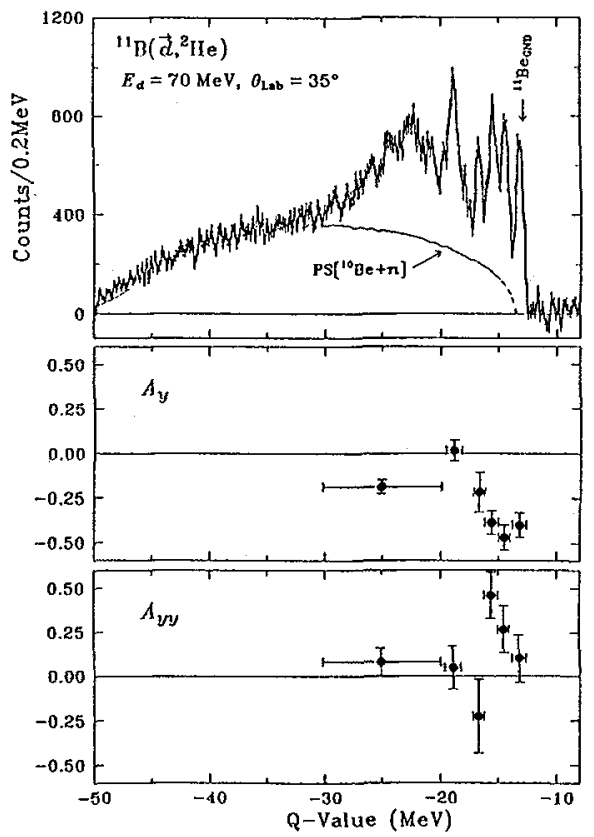

Figure 6: Energy spectrum and vector and tensor analyzing powers $\left(A_{y}, A_{y y}\right)$ for the ${ }^{11} \mathrm{~B}\left(\vec{d}^{2}, \mathrm{He}\right){ }^{11} \mathrm{Be}$ reaction at $70 \mathrm{MeV}$ at $\theta_{L a b}=35^{\circ}$.

2.3 Structure of neutron rich nucleus ${ }^{11} \mathrm{Be}$ studied by the ${ }^{11} \mathrm{~B}\left(\vec{d}^{2}, \mathrm{He}\right)$ reaction

Since the discovery of a neutron halo in large neutron-excess nuclei $,{ }^{11} \mathrm{Li},{ }^{11} \mathrm{Be},{ }^{14} \mathrm{Be} / 41 /$, a lot of interest has been placed on the nuclear structure of such nuclei. Little is known on the structure of ${ }^{11} \mathrm{Be}$. The ground state has an anomalous spin and parity of $\frac{1}{2}^{+}$. As for the excited state, spin and parity assignment has been made for only two states/42/. The shell model calculations by Fukunishi, Sagawa and Ohtsuka/43/ predict GamowTeller transitions $(\Delta \ell=0, \Delta S=1)$ in the low exited region $\left(E_{x} \leq 5 \mathrm{MeV}\right)$ and a lot of spin-flip dipole transitions $(\Delta \ell=1, \Delta S=1)$ forming a kind of spin-flip dipole giant resonance(SFDR) at $E_{x}=5-12 \mathrm{MeV}$. The SFDR is excited by the $r\left[Y_{1} \sigma\right]^{(1)} \tau_{+}$operator and exhausts $42 \%$ of the $S_{+}$sum rule value. Owing to the loosely bound neutron which is the origin of neutron halo the main strength of the SFDR is shifted down as much as $2 \mathrm{MeV}$. In order to get more structure information on ${ }^{11} \mathrm{Be}$ the ${ }^{11} \mathrm{~B}\left(\vec{d}_{3}^{2} \mathrm{He}\right)$ reaction has been employed at $70 \mathrm{MeV} / 40 /$. Here I show preliminary result.

Figure 6 shows the typical spectra for the cross sections and analyzing powers $\left(A_{y}, A_{y y}\right)$ at $\theta_{L a b}=35^{\circ}$. Several discrete peaks in the low excited region and broad bump at an excitation energy around $10 \mathrm{MeV}$ with a width of about $5 \mathrm{MeV}$ are clearly observed. The ground state seems to be not excited by this reaction. Angular distributions for representative states are shown in fig. 7 after subtracting a reasonable background as shown in fig.6. If we apply our empirical rule of $A_{y}$ to the transitions to the low lying states, it can be concluded that they are most probably due to the Gamow-Teller type transitions since their $A_{y}$ are large negative at forward angles. The spin-parity of the 

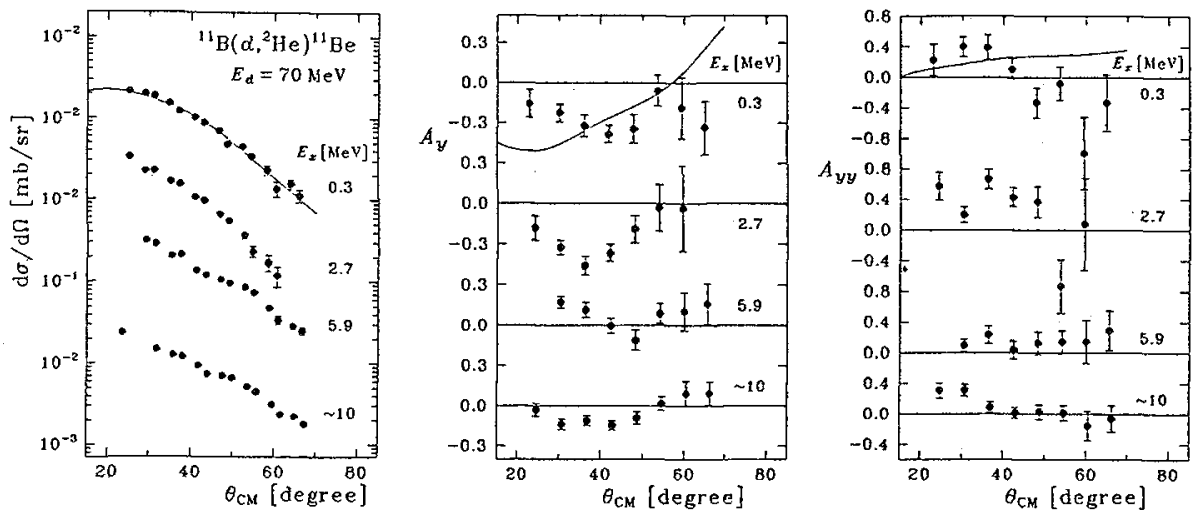

Figure 7: Differential cross section and vector and tensor analyzing powers $\left(A_{y}, A_{y y}\right)$ for the ${ }^{11} \mathrm{~B}\left(\vec{d},{ }^{2} \mathrm{He}\right){ }^{11} \mathrm{Be}$ reaction at $70 \mathrm{MeV}$ at $\theta_{L a b}=35^{\circ}$. The curve is the result of DWBA calculation.

first excited state at $E_{x}=0.32 \mathrm{MeV}$ is known to be $\frac{1}{2}^{-}$. Therefore the transition to the first excited state $\left(\frac{3}{2}^{-} \rightarrow \frac{1}{2}^{-}\right)$should be the Gamow-Teller type and indeed our empirical rule of $A_{y}$ nicely agrees with it. The DWBA calculation is performed for the first excited state with the shell model wave functions/43/ and an excellent fit for the cross section and resonable fits for the analyzing powers are obtained as shown in fig.7 as solid curves.

The broad bump observed at around $E_{x}=10 \mathrm{MeV}$ together with $5.9 \mathrm{MeV}$ state presumably corresponds to the SFDR predicted by the shell model calculation. This conclusion is supported by the evidence of a flatter shape of the angular distribution for these transitions indicating $\ell \geq 1$ compared to the low lying $\ell=0$ transitions(see fig.7).

It is clear that the $\left(\vec{d}^{2} \mathrm{He}\right)$ reaction is a powerful means to study the nuclear structure of the neutron rich nucleus and the analyzing power plays a major role in assigning Gamow-Teller type transitions.

\section{Summary}

In the first part I presented recent developments of the studies of transfer reactions by using polarized beams by showing three examples:

i) occupation probabilities around ${ }^{40} \mathrm{Ca}$,

ii) PTCs and the deuteron D-state amplitude and

iii) $(d, p)$ reaction and the quasi-ADWA.

Obvious merits of measuring polarization observables in the course of these studies are presented.

The transfer reaction which is important in its own right has been providing basic and valuable information for many years and it is still more expanding to various fields. However more efforts are demanded to establish further the transfer reaction as 
a quantitative probe to provide precise data.

In the second part I have shown the new development of the deuteron induced chargeexchange reactions, namely $\left(\vec{d}, d_{s=0}\right)$ and $\left(\vec{d}_{,}^{2} \mathrm{He}\right)$ reactions, which exclusively excite spinand isospin-flip transitions. The reaction mechanism has been studied to examine the validity of FSI treatment and then these reactions have been applied to many nuclei to investigate nuclear structures. As an example the study of the ${ }^{11} \mathrm{~B}\left(\vec{d}^{2} \mathrm{He}\right){ }^{11} \mathrm{Be}$ reaction is presented to show the ability of identifying the Gamow-Teller and the SFDR transitions where the effect due to the loosely bound neutron might appear.

Up to now the $\left(\vec{d}_{,}^{2} \mathrm{He}\right)$ reaction is studied at RCNP with a conventional SSD counter array and at SATURNE with an existing magnetic spectrograph SPES4. Their effective solid angles $\left(\Omega_{e f f}\right)^{1}$ are $1.2 \mathrm{msr}$ at $E_{d}=70 \mathrm{MeV}$ and $0.4 \mathrm{msr}$ at $E_{d}=2 \mathrm{GeV}$, respectively. Second generation magnetic spectrographs are being built at RIKEN/44/ and at Texas $\mathrm{A} \& \mathrm{M} / 45 /$. They are designed to detect the ${ }^{2} \mathrm{He}$ "particle"s from the beginning so that having large effective solid angles, $5.5 \mathrm{msr}$ at $E_{d}=270 \mathrm{MeV}$ (RIKEN) and $4 \mathrm{msr}$ at 160 $\mathrm{MeV}$ (Texas A\&M), respectively. Owing to this large solid angle and to the better energy resolution it is expected to yield high quality $\left(d,{ }^{2} \mathrm{He}\right)$ data in near future. The next conference on the Polarization Phenomena in Nuclear Physics will be flooded with these high quality data hopefully with new exciting physics.

\section{Acknowledgements}

The author wishes to thank to Professors M. Matoba, S. Nishizaki, K. Sagara, R.C. Johnson, and E.J. Stephenson for their discussions and help in preparing the first part of this talk. He is also grateful to all his colleagues who actively participated in the study of deuteron induced charge-exchange reactions. He thanks to Professor M. Kondo for his encouragement. Lastly but not least the author gratefully thanks to Dr. H. Okamura for his fruitful discussions and his help in preparing the second part of this talk.

\section{References}

/ 1/ V.R. Pandharipande,C.N. Papanicolas and J. Wambach, Phys. Rev. Lett. 53 (1984) 445.

/ 2/. M. Jaminon,C. Mahaux and H. Ngo, Nucl. Phys. A440 (1985) 228; Nucl. phys. A452 (1986) 445.

/ 3/ F.J. Eckle et al., Phys. Rev. C39 (1989) 1662.

/ 4/ F.J. Eckle et al., Nucl. Phys. A506 (1989) 1662.

/ 5/ M. Matoba, private communication.

/6/ M. Matoba et al., Phys. Rev. C39 (1989) 1658.

/ 7 / H. Ikegami et al., Nucl. Instrum. Methods 175 (1980) 33.

/ 8/ S. Platchkov et al., Phys. Rev. Lett. 61 (1988) 1465.

/ 9/ S. Nishizaki,S. Drozoz,J. Wambach, and J. Speth, Phys. Lett. B125 (1988) 231. /10/ For example, H. Lenske et al., contribution to this conference.

\footnotetext{
${ }^{1} \Omega_{e f f}$ is calculated based on the Migdal-Watson theory as defined in ref.36 but integration is made up to $\epsilon_{p p}=5 \mathrm{MeV}$.
} 
/11/ G.J. Kramer et al., Nucl. Phys. A477 (1988) 55.

/12/ G.J. Kramer, $\mathrm{PhD}$ thesis (Universiteit van Amsterdam),1990, unpublished.

113/ M.C. Padhakrishna, et al., Phys. Rev. C37 (1988) 66.

/14/ H. Clement,P. Grabmayr,H. Rohm, and G.J. Wagner, Phys. Lett. B183 (1987) 127.

/15/ P.K.A. de Witt Huberts, J. Phys. G16 (1990) 507.

/16/ M. Tanifuji and K. Yazaki, Prog. Theo. Phys. 40 (1968) 1023.

/17/ K. Sagara, Proceedings of the RIKEN symposium on LIGHT AND LIGHT-HEAVY ION REACTIONS, 1989 unpublished, p18.

/18/ K. Sagara et al., contributions to this conference.

/19/ R.V. Reid, Ann. Phys. 59 (1968) 441.

/20/ M. Tanifuji, private communication.

/21/ M. Kamimura et al., Prog. Theo. Phys. Supplement 89 (1986) 1.

/22/ R.C. Johnson, J. Phys. Soc. Jpn. 55 (1986) 7.

/23/ V.R. Cupps et al., Nucl. Phys. A469 (1987) 445.

/24/ E.J. Stephenson et al., Nucl. Phys. A469 (1987) 467.

/25/ R.C. Johnson et al., Nucl. Phys. A505 (1989) 26.

/26/ E.J. Stephenson et al., preprint 1990.

/27/ For example, "Spin Observables of Nuclear Probes", ed. by C.J. Horowitz et al.,1988, Plenum Press.

/28/ D.V. Bugg and C. Wilkin, Nucl. Phys. A 467 (1987) 575,

C. Wilkin and D.V. Bugg, Phys. Lett. 154B (1985) 243.

/29/ J. Delorme, invited talk.

/30/ A.B. Migdal, Sov. JETP 1 (1955) 2.

/31/ K.M. Watson, Phys. Rev.. 88 (1952) 1163.

/32/ H. Okamura, PhD thesis, Kyoto University, 1989, unpublished.

/33/ H. Okamura et al. Phys. Lett. 227B (1989) 204.

/34/ T. Motobayashi et al., Phys. Rev. C34 (1986) 2365.

/35/ T. Noro et al., J. Phys. Soc. Jpn. 55 (1986) 470.

/36/ T. Motobayashi et al., Nucl. Instrum. Methods. A271 (1988) 491.

/37/ T. Motobayashi et al., J.Phys. G: 14 (1988) L137.

/38/ A. Okihana et al., to be published. RCNP annual report $1988 \mathrm{p} 66$.

/39/ T. Motobayashi et al., Nucl. Phys. A481 (1988) 207.

/40/ H. Okamura, to be published.

/41/ I. Tanihata et al., Phys. Rev. Lett. 55 (1985) 2676.

/42/ F. Ajzenberg-Selove et al., Nucl. Phys. A506 (1990) 1.

/43/ N. Fukunishi, H. Sagawa and T. Ohtsuka, private communication.

/44/ H. Ohnuma et al., private communication.

/45/ A.C. Betker and C.A. Gagliardi, Nucl. Instrum. Methods. A281 (1989) 67. 